\title{
VARIATIONAL PRINCIPLES FOR LINEAR COUPLED THERMOELASTICITY*
}

\author{
BY \\ R. E. NICKELL $\dagger$ \\ AND \\ J. L. SACKMAN \\ University of California at Berkeley
}

\begin{abstract}
Several variational principles are derived for the initial-boundary-value problem of fully coupled linear thermoelasticity for an inhomogeneous, anisotropic continuum. A consistent set of field variables is employed and a method based on the Laplace transform is used to incorporate the initial conditions explicitly into the formulation. These principles lend themselves readily to numerical solutions based on an extended Ritz method.

1. Introduction. The application of variational methods for both the unified development of the theory and for the approximate solution of fully coupled initial-boundaryvalue problems in linear thermoelasticity is not new. As a starting point, the work of Biot [1] introduced a variational principle in terms of a pair of vector-valued primary variables, the displacement of a material point and a variable which he termed the entropy displacement. The Euler equations of the principle are the thermoelastic equations of motion and the corrected heat conduction equation. A generalization of Biot's principle [2] incorporated the additional primary variables of the stress tensor and a thermal dis-equilibrium force conjugate to the entropy displacement. Euler equations representing the linear thermoelastic stress-strain relationship, the Fourier heat conduction law, and a relationship between the temperature and the thermal gradient, as well as the aforementioned field equations, are products of that variational principle.

The most general variational statement of the coupled thermoelastic problem was made by Bao-Lian' Fu [3] and later by Ben-Amoz [4], both of whom obtain essentially the same Euler equations and natural boundary conditions. Both prescribe a boundary condition on the entropy displacement vector rather than on the heat flux vector, and Ben-Amoz obtains a sixth Euler equation, representing a relationship between the temperature and the thermal gradient.

Impetus for further development of variational principles for the coupled thermoelastic problem is suggested by recent work of Gurtin [5], [6]. In these treatments of linear elastodynamics and transient heat conduction Gurtin, utilizing the operational methods of Mikusinski [7], explicitly introduces the initial conditions appropriate to the problem into the field equations and governing functionals, and derives alternate characterizations of the problems. The following work represents an extension of these concepts to the field theory of linear coupled thermoelasticity, and an attempt to remove
\end{abstract}

\footnotetext{
*Received December 12, 1966. This research was supported in part by the U. S. Army Research Office-Durham, North Carolina, and the National Science Foundation.

†Currently, Research Engineer, Rohm \& Hass Company, Huntsville, Alabama.
} 
a lack of consistency (present in the existing variational formulations) in the choice of the field variables and boundary conditions.

2. Notation. Mathematical preliminaries. The notation and format used by Gurtin [5] will largely be employed here. Therefore the standard indicial system, with Greek and Latin subscripts ranging over the integral values $(1,2)$ and $(1,2,3)$ respectively, is used throughout. The reference frame is Cartesian, repeated subscripts imply summation, and Kronecker's delta is denoted by $\delta_{i j}$. Parenthetical superscripts, as well as superposed dots, indicate the order of time differentiation, and subscripts preceded by a comma denote space differentiation with respect to the Cartesian coordinates. Parentheses about a pair of free subscripts will signify the symmetric part of the tensor with respect to those subscripts.

A region $\bar{V}$ shall denote the closure of an open, bounded, connected set contained in the three-dimensional Euclidean space $E$. The boundary of $\bar{V}$ we denote by $S$, being the union of a finite number of nonintersecting closed regular surfaces. The term regular surface is used in the sense of Kellogg [8]. The interior of $\bar{V}$ is $V$, and $\mathbf{n}$ is the outward unit normal vector to $S . S_{\alpha}$ and $\S_{\alpha}$ are a dual system of complementary regular subsets of $S$, where $\bar{S}_{\alpha}$ and $\bar{S}_{\alpha}$ denote the closures of $S_{\alpha}$ and $S_{\alpha}$, respectively.

The domain of definition for all functions of the position vector $\mathrm{x}$ and the time $t$ is the set which is the Cartesian product of the region of space $\bar{V}$ and the interval of time $[0, \infty)$ denoted by $\bar{V} \times[0, \infty)$.

The values of a function $f(\mathbf{x}, t)$ and its derivatives are defined on the boundary of the domain of definition $\bar{V} \times[0, \infty)$ by

$$
f_{, i j \ldots k}^{(n)}\left(\mathbf{x}_{0}, t_{0}\right)=\lim _{(\mathbf{x}, t) \rightarrow\left(\mathbf{x}_{0}, t_{0}\right)} f_{, i j \ldots k}^{(n)}(\mathbf{x}, t),
$$

where $\mathbf{x}_{0} \in \bar{V}, t_{0}=0$ or $\mathbf{x}_{0} \in S, t_{0} \in[0, \infty)$. We say that the function $f$ is in the function class $C^{M, N}$ if and only if it is defined on $\bar{V} \times[0, \infty)$, and all of the functions

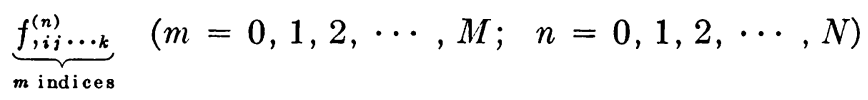

exist and are continuous on $\bar{V} \times[0, \infty)$.

A point $\mathbf{x} \in S$, or a point $(\mathbf{x}, t) \in S \times[0, \infty)$, such that $\mathbf{n}$ is continuous at $\mathbf{x}$, will be termed a regular point. A function $f$ will be said to be piecewise regular on $\bar{S}_{\alpha} \times[0, \infty)$ if 'and only if $f$ is piecewise continuous on $\bar{S}_{\alpha} \times[0, \infty)$, and every regular point of that region is a point of continuity of $f$. Two piecewise regular functions defined on $\bar{S}_{\alpha} \times$ $[0, \infty)$ are said to be equal if and only if they are equal at every regular point $(\mathbf{x}, t) \in \bar{S}_{\alpha} \times[0, \infty)$.

Let $f$ and $g$ be functions of space and time defined on $\bar{V} \times[0, \infty)$ such that both are continuous on $[0, \infty)$ for each $\mathbf{x} \in V$. The convolution of $f$ and $g$ is given by

$$
[f * g](\mathbf{x}, t)=\int_{0}^{t} f(\mathbf{x}, t-\tau) g(\mathbf{x}, \tau) d \tau, \quad(\mathbf{x}, t) \in \bar{V} \times[0, \infty),
$$

having the well-known properties:

(b) $g *(f * h)=(g * f) * h=g * f * h$,

(c) $g *(f+h)=g * f+g * h$. 
A corollary of Titchmarsh's theorem [9] will prove useful in later work and is introduced in the form

$$
f * g=0 \text { implies either } f=0 \text { or } g=0 .
$$

The term functional will be used to identify a real-valued function whose domain is a subset of a linear space. If $L$ is a linear space, $K$ a subset of $L$, and $\Omega\{\cdot\}$ a functional defined on $K$, then for

$$
R, \tilde{R} \in L, \quad R+\lambda \tilde{R} \in K \text { for every real } \lambda,
$$

formally define the notation

$$
\delta_{\tilde{R}} \Omega\{R\}=\left.\frac{d}{d \lambda} \Omega\{R+\lambda \tilde{R}\}\right|_{\lambda=0} .
$$

The variation of $\Omega\{\cdot\}$ is zero at $R$ over $K$ and is written

$$
\delta \Omega\{R\}=0 \text { over } K,
$$

if and only if $\delta_{\tilde{R}} \Omega\{R\}$ exists and equals zero for every choice of $\tilde{R}$ consistent with (2.6).

Three lemmas which are analogous to the fundamental lemma of the calculus of variations, and which have been proved by Gurtin [5] are now stated. Based on these lemmas we also state a corollary which will be needed in the subsequent development.

2.1. Lemma. Let $f$ be a continuous function on $\bar{V} \times[0, \infty)$, and suppose

$$
\int_{V}[f * g](\mathbf{x}, t) d V=0 \quad(0 \leqq t<\infty)
$$

for every $g \in C^{\infty, \infty}$ which, together with its space derivatives, vanishes on $S \times[0, \infty)$. Then

$$
f=0 \text { on } \bar{V} \times[0, \infty) .
$$

2.2 Lemma. Let $f$ be a piecewise regular function on $\overline{S_{2}} \times[0, \infty)$, and suppose,

$$
\int_{S_{2}}[f * g](\mathbf{x}, t) d S=0 \quad(0 \leqq t<\infty)
$$

for every $g \in C^{\infty, \infty}$ that vanishes on $S_{1} \times[0, \infty)$. Then

$$
f=0 \text { on } \bar{S}_{2} \times[0, \infty) \text {. }
$$

2.3 Lemмa. Let $f_{i}$ be continuous on $\bar{S}_{1} \times[0, \infty)$, and suppose

$$
\int_{S_{1}}\left[f_{i} *\left(g_{i j} n_{j}\right)\right](\mathrm{x}, t) d S=0 \quad(0 \leqq t<\infty)
$$

for every $g_{i j} \in C^{\infty, \infty}$ which, together with all its space derivatives, vanishes on $S_{2} \times[0, \infty)$ and which has the property

$$
g_{i j}=g_{i i}
$$

Then

$$
f_{i}=0 \text { on } \bar{S}_{1} \times[0, \infty) .
$$

A corollary of Lemma 2.3 is: 
2.4 Corollary. Let $f$ be continuous on $\bar{s}_{1} \times[0, \infty)$, and suppose

$$
\int_{S_{1}}\left[f *\left(g_{i} n_{i}\right)\right](\mathbf{x}, t) d S=0 \quad(0 \leqq t<\infty)
$$

for every $g_{i} \times C^{\infty, \infty}$ which, together with its space derivatives, vanishes on $S_{2} \times[0, \infty)$. Then

$$
f=0 \text { on } \bar{s}_{1} \times[0, \infty) .
$$

3. The initial-boundary-value problem with mixed boundary conditions. The fundamental system of field equations for linear coupled thermoelasticity, characterizing inhomogeneous and anisotropic solids, is now stated for reference. Let $\bar{V}$ be the region of space occupied by the solid and let $\bar{V} \times[0, \infty)$ denote the domain of definition for all functions of position and time.

Let $u_{i}(\mathbf{x}, t), e_{i j}(\mathbf{x}, t), \tau_{i j}(\mathbf{x}, t), F_{i}(\mathbf{x}, t), q_{i}(\mathbf{x}, t)$, and $\vartheta_{i}(\mathbf{x}, t)$, in this order, represent the Cartesian components of the displacement vector $\mathbf{u}(\mathbf{x}, t)$, the infinitesimal strain tensor $\mathbf{e}(\mathbf{x}, t)$, the stress tensor $\tau(\mathbf{x}, t)$, the body force vector $\mathbf{F}(\mathbf{x}, t)$, the heat flux vector $\mathbf{q}(\mathbf{x}, t)$, and the thermal gradient vector $\boldsymbol{\vartheta}(\mathbf{x}, t)$. Also let $\theta(\mathbf{x}, t)$ denote the temperature above a quiescent reference state $T_{0}$ (a constant absolute temperature), and let $\eta(\mathbf{x}, t)$ and $H(\mathrm{x}, t)$, respectively, be the specific entropy per unit mass and the rate of internal heat generation per unit volume within the solid.

Then the strain-displacement and thermal gradient-temperature relations are

$$
e_{i j}=\frac{1}{2}\left(u_{i, i}+u_{i, i}\right)=u_{(i, i)}
$$

and

$$
\vartheta_{i}=\theta_{, i} \text { on } V \times[0, \infty) .
$$

The equations of motion and of energy are, respectively,

$$
\tau_{i, i}+F_{i}=\rho \ddot{u}_{i}, \quad \tau_{i j}=\tau_{j i},
$$

and

$$
q_{i, i}+\rho T_{0} \dot{\eta}=H \quad \text { on } \quad V \times[0, \infty),
$$

where $\rho(\mathbf{x})$ is the mass density of the solid. Then introduce $c_{i j k l}(\mathbf{x}), k_{i j}(\mathbf{x}), \alpha_{i j}(\mathbf{x}), C_{0}(\mathbf{x})$, and $C_{\tau}(\mathbf{x})$ as, respectively, the components of the isothermal elasticity tensor $\mathbf{c}(\mathbf{x})$, the thermal conductivity tensor $\mathbf{k}(\mathbf{x})$, the thermal expansion tensor $\boldsymbol{\alpha}(\mathbf{x})$, and the scalar specific heats for zero deformation and for zero stress. The three sets of tensor components, $\chi_{i j k l}(\mathbf{x}), \lambda_{i j}(\mathbf{x})$, and $\beta_{i j}(\mathrm{x})$, will be termed the components of the isothermal compliance tensor $\chi(x)$, the thermal resistivity tensor $\lambda(\mathbf{x})$, and the thermoelasticity tensor $\boldsymbol{\beta}(\mathbf{x})$. These quantities are related through

$$
\begin{aligned}
c_{i j k l} \chi_{k l m n} & =\delta_{i m} \delta_{i n}, \\
k_{i m} \lambda_{m i} & =\delta_{i j}, \\
\beta_{i j} & =c_{i j k l} \alpha_{k l}, \\
C_{\bullet} & =C_{\tau}-\alpha_{i j} \beta_{i j} T_{0} / \rho \text { on } V,
\end{aligned}
$$

and satisfy the symmetry conditions 


$$
\begin{aligned}
c_{i j k l} & =c_{i i k l}=c_{k l i j}, \\
\chi_{i j k l} & =\chi_{i i k l}=\chi_{k l i j}, \\
k_{i j} & =k_{j i}, \\
\lambda_{i j} & =\lambda_{i i}, \\
\alpha_{i j} & =\alpha_{i i}, \\
\beta_{i j} & =\beta_{i i} \text { on } V .
\end{aligned}
$$

The constitutive relations and the equation of state are then

$$
\begin{aligned}
\tau_{i j} & =c_{i j k l} e_{k l}-\beta_{i j} \theta, \\
q_{i} & =-k_{i j} \vartheta_{i},
\end{aligned}
$$

and

$$
\rho T_{0} \eta=\rho C_{0} \theta+\beta_{i j} T_{0} e_{i j} \quad \text { on } \quad V \times[0, \infty),
$$

or, alternatively,

$$
\begin{aligned}
e_{i j} & =\chi_{i j k l} \tau_{k l}+\alpha_{i j} \theta, \\
\vartheta_{i} & =-\lambda_{i j} q_{i}
\end{aligned}
$$

and

$$
\rho T_{0} \eta=\rho C_{\tau} \theta+\alpha_{i i} T_{0} \tau_{i i} \quad \text { on } \quad V \times[0, \infty) .
$$

Associated with this system of field equations are the initial conditions

$$
\begin{aligned}
& u_{i}(\mathbf{x}, 0)=d_{i}(\mathbf{x}), \\
& \dot{u}_{i}(\mathbf{x}, 0)=v_{i}(\mathbf{x}),
\end{aligned}
$$

and

$$
\theta(\mathrm{x}, 0)=\theta_{0}(\mathrm{x}) \text { on } \bar{V}
$$

the displacement boundary conditions

$$
u_{i}=\hat{u}_{i} \quad \text { on } \quad S_{1} \times[0, \infty),
$$

the traction boundary conditions

$$
T_{i}=\tau_{i j} n_{i}=\hat{T}_{i} \quad \text { on } \quad S_{2} \times[0, \infty),
$$

the temperature boundary condition

$$
\theta=\hat{\theta} \quad \text { on } \quad S_{1} \times[0, \infty),
$$

and the heat flux boundary condition

$$
Q=q_{i} n_{i}=\hat{Q} \quad \text { on } \quad \delta_{2} \times[0, \infty) .
$$

Here $\mathbf{d}, \mathbf{v}$, and $\theta_{0}$ are the prescribed initial displacements, initial velocities, and initial temperature distribution, while $\hat{\mathfrak{u}}, \hat{\mathrm{T}}, \hat{\theta}$, and $\hat{Q}$ are the given surface displacements, tractions, temperature, and normal heat flow. 
It should be noted that the boundary conditions prescribed above may be generalized to include mixed-mixed conditions for tractions and displacements, as well as the more general "radiation" type boundary conditions, such as the elastically supported surface and the thermal convection boundary layer.

Analogous to the work of Gurtin [5], the smoothness requirements and other regularity assumptions on the ascribable functions are introduced as hypotheses on the data:

(i) $\rho>0$ is continuously differentiable on $\bar{V}$;

(ii) $\mathbf{c}$ and $x$ are continuously differentiable on $\bar{V}$ and satisfy (3.3a), (3.4a), and

(iii) $\quad C_{c}>0$ and $C_{\tau}>0$ are continuously differentiable on $\bar{V}$ and satisfy (3.3d);

(iv) $\mathbf{k}, \boldsymbol{\alpha}, \lambda$, and $\boldsymbol{\beta}$ are continuously differentiable on $\bar{V}$ and satisfy (3.3b), (3.3c), (3.4c), (3.4d), (3.4e), and (3.4f);

(v) $\mathrm{d}$ is continuously differentiable on $\bar{V}$;

(vi) $\nabla$ and $\theta_{0}$ are continuous on $\bar{V}$;

(vii) $\mathbf{F}$ and $H$ are continuously differentiable on $\bar{V} \times[0, \infty)$;

(viii) $\hat{\mathbf{u}}$ and $\hat{\theta}$ are continuous on $\bar{S}_{1} \times[0, \infty)$ and $\bar{s}_{1} \times[0, \infty)$, respectively;

(ix) $\hat{\mathrm{T}}$ and $\hat{Q}$ are piecewise continuous on $\bar{S}_{2} \times[0, \infty)$ and $\bar{S}_{2} \times[0, \infty)$, respectively.

With the specifications on the data cited above the mixed problem consists of finding a set of functions $[\mathbf{u}, \mathbf{e}, \tau, \theta, \eta, \mathbf{q}, \boldsymbol{\vartheta}]$ on $\bar{V} \times[0, \infty)$ which satisfies the field equations (3.1), (3.2), (3.5), the initial conditions (3.7), and the boundary conditions (3.8).

The ultimate goal is to transform the foregoing statement of the mixed initialboundary-value problem into an equivalent variational formulation. To accomplish this, it is convenient to define an admissible state, and then, in terms of admissible states, a solution of the mixed problem. An admissible state, denoted as $R=[\mathbf{u}, \mathbf{e}, \tau, \theta, \eta, \mathbf{q}, \boldsymbol{\vartheta}]$, is an ordered array of functions $\mathfrak{u}, \mathbf{e}, \tau, \theta, \eta, \mathbf{q}, \boldsymbol{\vartheta}$ defined on $\bar{V} \times[0, \infty)$ with the properties:

(a) $\quad u_{i} \in C^{1,2}, \quad e_{i j} \in C^{0,0}, \quad \tau_{i j} \in C^{1,0}, \quad \theta \in C^{1,0}$,

$$
\eta \in C^{0,1}, \quad q_{i} \in C^{1,0}, \quad \vartheta_{i} \in C^{0,0} ;
$$

(b) $e_{i j}=e_{i i}, \quad \tau_{i j}=\tau_{j i}$ on $\bar{V} \times[0, \infty)$.

Addition of states and multiplication of a state by a scalar are defined by

$$
R+\tilde{R}=[\mathbf{u}+\tilde{\mathbf{u}}, \mathbf{e}+\tilde{\mathbf{e}}, \tau+\tilde{\tau}, \theta+\tilde{\theta}, \eta+\tilde{\eta}, \mathbf{q}+\tilde{\mathbf{q}}, \boldsymbol{\vartheta}+\tilde{\boldsymbol{\vartheta}}]
$$

and

$$
\lambda R=[\lambda \mathfrak{u}, \lambda \mathbf{e}, \lambda \tau, \lambda \theta, \lambda \eta, \lambda \mathbf{q}, \lambda \boldsymbol{\vartheta}] .
$$

In this way the set of all admissible states is a linear space.

A solution of the mixed problem is now defined as an admissible state $R=[\mathbf{u}, \mathbf{e}, \tau$, $\theta, \eta, \mathbf{q}, \boldsymbol{\vartheta}]$ which satisfies the field equations (3.1), (3.2), (3.5), the initial conditions (3.7) and the boundary conditions (3.8).

4. Alternative formulations. Since a major motive for the recasting of the initialboundary-value problem of coupled thermoelasticity is to incorporate the initial conditions explicitly into the field equations and into the functionals which arise in the variational formulations, it is convenient to consider the Laplace transformation of Eqs. (3.2). Using (3.5c) and (3.7) 


$$
\bar{\tau}_{i j, i}+\bar{F}_{i}+\rho s d_{i}+\rho v_{i}=\rho s^{2} \bar{u}_{i}
$$

and

$$
\bar{q}_{i, i}-\rho C_{e} \theta_{0}-\beta_{i j} T_{0} d_{i, j}+\rho T_{0} s \bar{\eta}=\bar{H} .
$$

where a superimposed bar denotes the transformed function and $s$ is the transformation parameter. Solving (4.1) for $\bar{u}_{i}$ and $\bar{\eta}$, then applying the inverse transformation, yields

$$
\rho u_{i}=g * \tau_{i j, i}+f_{i}
$$

and

$$
\rho T_{0} \eta=h-g^{\prime} * q_{i, i},
$$

where the functions $g, g^{\prime}, f_{i}$, and $h$ are defined to be

$$
\begin{gathered}
g(t)=t, \quad g^{\prime}(t)=1 \quad(0 \leqq t<\infty), \\
f_{i}(\mathbf{x}, t)=\left[g * F_{i}\right](\mathbf{x}, t)+\rho(\mathbf{x})\left[t_{i}(\mathbf{x})+d_{i}(\mathbf{x})\right],
\end{gathered}
$$

and

$$
h(\mathbf{x}, t)=\left[g^{\prime} * H\right](\mathbf{x}, t)+\rho(\mathbf{x}) C_{e}(\mathbf{x}) \theta_{0}(\mathbf{x})+T_{0} \beta_{i j}(\mathbf{x}) d_{i, j}(\mathbf{x}), \quad(\mathbf{x}, t) \in V \times[0 . \infty) .
$$

The functions $f_{i}$ and $h$ are completely described by information relevant to the mixed problem.

With these results in mind alternative formulations of the problem can easily be made.

4.1 Theorem. Let $u_{i} \in C^{0,2}, \tau_{i j} \in C^{1,0}$, and suppose $\tau_{i j}=\tau_{i i}$. Then $u_{i}, \tau_{i j}$ satisfy the equations of motion (3.2a) as well as the initial conditions (3.7a) and (3.7b) if and only if

$$
g * \tau_{i i, j}+f_{i}=\rho u_{i} \text { on } \quad V \times[0, \infty) .
$$

The proof of this theorem has been given by Gurtin [5].

4.2. Theorem. Let $\eta \in C^{0,1}, q_{i} \in C^{1,0}$, and suppose the equation of state (3.5c) holds for $t=0$. Then $\eta$ and $q_{i}$ satisfy the energy equation (3.2b) as well as the initial conditions $(3.7 \mathrm{c})$ if and only if

$$
h-g^{\prime} * q_{i, i}=\rho T_{0} \eta \quad \text { on } \quad V \times[0, \infty) .
$$

Proof. Suppose (3.2b), (3.7c), and (3.5c) (for $t=0$ ) hold. Then (4.3a) implies

$$
\begin{aligned}
{\left[g^{\prime} *\left(H-q_{i, i}\right)\right](\mathbf{x}, t) } & =\rho(\mathbf{x}) T_{0} \int_{0}^{t} \dot{\eta}(\mathbf{x}, \tau) d \tau \\
& =\rho(\mathbf{x}) T_{0} \eta(\mathbf{x}, t)-\rho(\mathbf{x}) T_{0} \eta(\mathbf{x}, 0) \\
& =\rho(\mathbf{x}) T_{0} \eta(\mathbf{x}, t)-\rho(\mathbf{x}) C_{e}(\mathbf{x}) \theta_{0}(\mathbf{x})-T_{0} \beta_{i j}(\mathbf{x}) d_{i, j}(\mathbf{x}) .
\end{aligned}
$$

Thus, and by (4.3c), equation (4.5) is met. Conversely, suppose (4.5) holds. Then, by reversing the argument, and utilizing the definitions (4.3a) and (4.3c), it is directly verified that $\eta, q_{i}$ meet (3.2b). Since (3.5c) (for $\left.t=0\right)$, (4.3a), (4.3c), (4.5) imply (3.7c), the proof is complete. 
4.3. Theorem. Let $R=[\mathbf{u}, \mathbf{e}, \tau, \theta, \eta, \mathbf{q}, \boldsymbol{\vartheta}]$ be an admissible state. Then $R$ is a solution to the mixed problem of coupled thermoelasticity if and only if it meets the field equations (3.1), (3.5), (4.4), (4.5), and the boundary conditions (3.8).

This result is a trivial consequence of Theorems 4.1 and 4.2.

As a result of this theorem, an alternative characterization of the solution to the mixed problem of coupled thermoelasticity has been developed such that the initial conditions are explicitly incorporated into two of the field equations.

By a displacement and temperature field corresponding to a solution of the mixed problem is meant an ordered pair $[\mathbf{u}, \theta]$ made up of a vector-valued function $\mathbf{u}$ and a scalar-valued function $\theta$ such that there exist functions $\mathbf{e}, \tau, \eta, \mathbf{q}, \boldsymbol{\theta}$ with the property that $[\mathbf{u}, \mathbf{e}, \tau$, $\theta, \eta, \mathbf{q}, \boldsymbol{\vartheta}]$ is a solution to the mixed problem.

4.4. Theorem. Let $u_{i} \in C^{2,2}$ and $\theta \in C^{2,0}$. Then $\mathfrak{u}, \theta$ is a displacement and temperature field corresponding to a solution of the mixed problem of coupled thermoelasticity if and only if

$$
g *\left(c_{i j k l} u_{k, l}-\beta_{i j} \theta\right)_{, i}+f_{i}=\rho u_{i}
$$

and

$$
\begin{aligned}
g^{\prime} *\left(k_{i j} \theta_{i j}\right)_{i}+h & =\rho C_{e} \theta+T_{0} \beta_{i j} u_{i, i} \quad \text { on } \quad V \times[0, \infty), \\
u_{i} & =\hat{u}_{i} \text { on } \quad S_{1} \times[0, \infty), \\
\left(c_{i j k l} u_{k, l}-\beta_{i j} \theta\right) n_{i} & =\hat{T}_{i} \text { on } \quad S_{2} \times[0, \infty), \\
\theta & =\hat{\theta} \text { on } \delta_{1} \times[0, \infty),
\end{aligned}
$$

and

$$
-k_{i j} \theta,{ }_{i} n_{i}=\hat{Q} \quad \text { on } \quad \delta_{2} \times[0, \infty) .
$$

Proof. First suppose $\mathfrak{u}$ and $\theta$ meet Eqs. (4.7). Define e and $\boldsymbol{\vartheta}$ through (3.1) and $\tau, \mathbf{q}, \eta$ through (3.5). Then, and because of (3.4), the boundary conditions (3.8) are met. Further (4.7a), (4.7b), (3.1), (3.4), (3.5) imply (4.4), (4.5). Thus, and by Theorem 4.3, [u, $\mathbf{e}, \tau, \theta, \eta, \mathbf{q}, \boldsymbol{\vartheta}]$ is a solution to the mixed problem. Conversely, (3.1), (3.4), (3.5), (3.8), (4.4), (4.5) imply (4.7) and the proof is therefore complete.

By a stress and heat flux field corresponding to a solution of the mixed problem is meant an ordered pair $[\tau, q]$ made up of a symmetric second-order tensor-valued function $\tau$ and a vector-valued function $\mathbf{q}$ such that there exist functions $\mathfrak{u}, \mathbf{e}, \theta, \eta, \boldsymbol{\vartheta}$ with the property that $[\mathbf{u}, \mathbf{e}, \boldsymbol{\tau}, \theta, \eta, \mathbf{q}, \boldsymbol{\vartheta}]$ is a solution of the mixed problem.

4.5. Theonem. Let $\tau_{i j} \in C^{2,0}$ and $q_{i} \in C^{2,0}$ with $\tau_{i j}=\tau_{i i}$. Then $\tau$, $\mathbf{q}$ is a stress and heat flux field corresponding to the solution of the mixed problem of couplcd thermoelasticity if and only if

$$
\left[\frac{1}{\rho}\left(g * \tau_{(i m, m}+f_{(i}\right)\right]_{, i)}-\frac{\alpha_{i, j}^{\prime}}{T_{0}^{\prime}}\left(h-g^{\prime} * q_{m, m}\right)=\chi_{i j k l}^{\prime} \tau_{k l l}
$$

and

$$
\left[\alpha_{k l}^{\prime} \tau_{k l}-\frac{1}{\rho C_{\tau}}\left(h-g^{\prime} * q_{m, m}\right)\right]_{, i}=\lambda_{i j} q_{i} \text { on } \quad V \times[0, \infty),
$$




$$
\begin{array}{rlll}
\frac{1}{\rho}\left(g * \tau_{i j, j}+f_{i}\right) & =\hat{u}_{i} & \text { on } & S_{1} \times[0, \infty), \\
\tau_{i j} n_{j}=T_{i} & =\hat{T}_{i} & \text { on } & S_{2} \times[0, \infty), \\
\frac{1}{\rho C_{\tau}}\left(h-g^{\prime} * q_{m, m}\right)-\alpha_{i j}^{\prime} \tau_{i j} & =\hat{\theta} & \text { on } & S_{1} \times[0, \infty),
\end{array}
$$

and

$$
q_{i} n_{i}=Q=\hat{Q} \quad \text { on } \quad \delta_{2} \times[0, \infty),
$$

where

$$
\alpha_{i j}^{\prime}(\mathrm{x})=\frac{T_{0} \alpha_{i j}(\mathrm{x})}{\rho(\mathrm{x}) C_{\tau}(\mathrm{x})}, \quad \chi_{i j k l}^{\prime}(\mathrm{x})=\chi_{i j k l}(\mathrm{x})-\alpha_{i j}(\mathrm{x}) \alpha_{k l}^{\prime}(\mathrm{x}) .
$$

Proof First suppose $\tau$ and $\mathbf{q}$ meet Eqs. (4.8). Define $\mathbf{u}$ from (4.4), $\eta$ from (4.5), $\theta$ from (3.6c), e from (3.6a), and $\vartheta$ from (3.6b). Then (4.8a), (4.8b), (4.8c), (4.8e), (4.9), (3.6), (4.4), (4.5) imply (3.1), (3.8a), (3.8c). Finally (3.5) hold by virtue of (3.3), (3.6). Thus, and by Theorem 4.3 , [u, e, $\tau, \theta, \eta, \mathbf{q}, \boldsymbol{\vartheta}]$ is a solution of the mixed problem. Conversely, (3.1), (3.3), (3.5), (4.4), (4.5), (3.8) imply (4.8) and the proof is complete.

The fact that the behavior of the stresses in linear elastodynamics can be characterized by a single tensorial field equation, similar in form to (4.8a), is due to Ignaczak [10]. Here, by (4.8a), (4.8b), this has been extended to the linear theory of dynamic, coupled thermoelasticity.

5. Variational principles characterizing coupled thermoelasticity. The most general variational principle to be considered here will be one in which the admissible states (which constitute a linear space) are not required to meet any of the field equations, initial conditions, or boundary conditions. A number of less general principles can also be derived, depending on the extent to which certain of the requirements are taken to be identically satisfied by the admissible states. Three principles will be formulated here, corresponding roughly to the elasto-static variational principles of $\mathrm{Hu}-\mathrm{Washizu}$ [11], [12], Hellinger-Reissner [13], [14], and minimum potential energy [15].

For consequent use, recall from Eqs. (4.3) that

$$
\begin{aligned}
g(t) & =t, \quad g^{\prime}(t)=1 \quad(0 \leqq t<\infty), \\
f_{i}(\mathbf{x}, t) & =\left[g * F_{i}\right](\mathbf{x}, t)+\rho(\mathbf{x})\left[t v_{i}(\mathbf{x})+d_{i}(\mathbf{x})\right],
\end{aligned}
$$

and

$$
h(\mathbf{x}, t)=\left[g^{\prime} * H\right](\mathbf{x}, t)+\rho(\mathbf{x}) C_{e}(\mathrm{x}) \theta_{0}(\mathbf{x})+T_{0} \beta_{i j}(\mathrm{x}) d_{i, j}(\mathrm{x}), \quad(\mathbf{x}, t) \in \bar{V} \times[0, \infty) .
$$

Also $\mathbf{T}$ and $\tilde{\mathbf{T}}$ shall be written for the traction vectors with components

$$
T_{i}=\tau_{i j} n_{i}, \quad \tilde{T}_{i}=\tilde{\tau}_{i j} n_{i},
$$

and $Q$ and $\widetilde{Q}$ for the normal heat fluxes given by

$$
Q=q_{i} n_{i}, \quad \widetilde{Q}=\tilde{q}_{i} n_{i} .
$$

5.1. Theorem. Let $K$ be the set of all admissible states. Let $R=[\mathfrak{u}, \mathbf{e}, \tau, \theta, \eta, \mathbf{q}, \boldsymbol{\vartheta}] \in K$, and for each $t \in[0, \infty)$ define the functional $\Omega_{t}\{\cdot\}$ on $K$ by 


$$
\begin{aligned}
\Omega_{t}\{R\}= & \frac{1}{2} \int_{V} c_{i j k l}^{\prime}(\mathbf{x})\left[g * e_{i j} * e_{k l}\right](\mathbf{x}, t) d V-\int_{V} \rho(\mathbf{x}) \beta_{i j}^{\prime}(\mathbf{x})\left[g * \eta * e_{i j}\right](\mathbf{x}, t) d V \\
& +\frac{1}{2} \int_{V} \frac{T_{0} \rho(\mathbf{x})}{C_{e}(\mathbf{x})}[g * \eta * \eta](\mathbf{x}, t) d V+\frac{1}{2} \int_{V} \rho(\mathbf{x})\left[u_{i} * u_{i}\right](\mathbf{x}, t) d V \\
& -\frac{1}{2} \int_{V} \frac{k_{i j}(\mathbf{x})}{T_{0}}\left[g * g^{\prime} * \vartheta_{i} * \vartheta_{i}\right](\mathbf{x}, t) d V-\int_{V}\left[g * \tau_{i j} * e_{i j}\right](\mathbf{x}, t) d V \\
& -\int_{V} \rho(\mathbf{x})[g * \eta * \theta](\mathbf{x}, t) d V-\int_{V} \frac{1}{T_{0}}\left[g * g^{\prime} * q_{i} * \vartheta_{i}\right](\mathbf{x}, t) d V \\
& -\int_{V}\left[\left(g * \tau_{i j, i}+f_{i}\right) * u_{i}\right](\mathbf{x}, t) d V-\int_{V} \frac{1}{T_{0}}\left[g *\left(g^{\prime} * q_{i, i}-h\right) * \theta\right](\mathbf{x}, t) d V \\
& +\int_{S_{1}}\left[g * T_{i} * \hat{u}_{i}\right](\mathbf{x}, t) d S+\int_{S,}\left[g *\left(T_{i}-\hat{T}_{i}\right) * u_{i}\right](\mathbf{x}, t) d S \\
& +\int_{S_{1}} \frac{1}{T_{0}}\left[g * g^{\prime} * Q * \hat{\theta}\right](\mathbf{x}, t) d S+\int_{s_{2}} \frac{1}{T_{0}}\left[g * g^{\prime} *(Q-\hat{Q}) * \theta\right](\mathbf{x}, t) d S,
\end{aligned}
$$

where

and

$$
\beta_{i j}^{\prime}(\mathbf{x})=\frac{T_{0}}{\rho(\mathbf{x}) C_{e}(\mathbf{x})} \beta_{i j}(\mathbf{x})
$$

Then

$$
c_{i j k l}^{\prime}(\mathbf{x})=c_{i j k l}(\mathbf{x})+\beta_{i j}(\mathbf{x}) \beta_{k l}^{\prime}(\mathbf{x}) .
$$

$$
\delta \Omega_{t}\{R\}=0 \quad \text { over } K \quad(0 \leqq t<\infty)
$$

if and only if $R$ is a solution of the mixed problem.

Proof. Let $\tilde{R}=[\tilde{\mathbf{u}}, \tilde{\mathbf{e}}, \tilde{\boldsymbol{\tau}}, \tilde{\theta}, \tilde{\eta}, \tilde{\mathbf{q}}, \tilde{\boldsymbol{\vartheta}}] \in K$, from which it follows that $R+\lambda \tilde{R} \in K$ for every scalar $\lambda$. Then, by (5.2), (2.7), (3.4), (5.1d), (5.1e), property (b) of admissible states, the properties of the convolution, and the divergence theorem,

$$
\begin{aligned}
\delta_{\tilde{R}} \Omega_{t}\{R\}= & \int_{V}\left[g *\left(c_{i j k l}^{\prime} e_{k l}-\rho \beta_{i j}^{\prime} \eta-\tau_{i j}\right) * \tilde{e}_{i j}\right](\mathbf{x}, t) d V \\
& +\int_{V} \rho(\mathbf{x})\left[g *\left(\frac{T_{0} \eta}{C_{e}}-\beta_{i j}^{\prime} e_{i j}-\theta\right) * \tilde{\eta}\right](\mathbf{x}, t) d V \\
& -\int_{V} \frac{1}{T_{0}}\left[g * g^{\prime} *\left(k_{i j} \vartheta_{i}+q_{i}\right) * \tilde{\vartheta}_{i}\right](\mathbf{x}, t) d V \\
& -\int_{V}\left[\left(g * \tau_{i, i}+f_{i}-\rho u_{i}\right) * \tilde{u}_{i}\right](\mathbf{x}, t) d V \\
& -\int_{V} \frac{1}{T_{0}}\left[g *\left(g^{\prime} * q_{i, i}-h+\rho T_{0} \eta\right) * \tilde{\theta}\right](\mathbf{x}, t) d V \\
& +\int_{V}\left[g *\left(u_{(i, i)}-e_{i j}\right) * \tilde{\tau}_{i j}\right](\mathbf{x}, t) d V \\
& +\int_{V} \frac{1}{T_{0}}\left[g * g^{\prime} *\left(\theta,,_{i}-\vartheta_{i}\right) * \tilde{q}_{i}\right](\mathbf{x}, t) d V+\int_{S_{\mathbf{z}}}\left[g *\left(\hat{u}_{i}-u_{i}\right) * \tilde{T}_{i}\right](\mathbf{x}, t) d S \\
& +\int_{S_{2}}\left[g *\left(T_{i}-\hat{T}_{i}\right) * \tilde{u}_{i}\right](\mathbf{x}, t) d S+\int_{s_{i}} \frac{1}{T_{0}}\left[g * g^{\prime} *(\hat{\theta}-\theta) * \tilde{Q}\right](\mathbf{x}, t) d S \\
& +\int_{s_{s}} \frac{1}{T_{0}}\left[g * g^{\prime} *(Q-\hat{Q}) * \tilde{\theta}\right](\mathbf{x}, t) d S \quad(0 \leqq t<\infty) .
\end{aligned}
$$


First suppose $R$ is a solution to the mixed problem. Then, by virtue of Theorem 4.3, (5.5) yields

$$
\delta_{\tilde{R}} \Omega_{t}\{R\}=0 \quad(0 \leqq t<\infty) \quad \text { for every } \quad \tilde{R} \in K,
$$

which implies (5.4).

Now examine the "only if" portion. It must be shown that $R \in K$ is a solution to the mixed problem whenever (5.6) holds. Choose $\tilde{R}=[\tilde{\mathbf{u}}, 0,0,0,0,0,0]$, and let $\tilde{\mathfrak{u}}$, together with all of its space derivatives, vanish on $S \times[0, \infty)$. Then, from (5.1), (5.5), (5.6), follows

$$
\int_{V}\left[\left(g * \tau_{i i, j}+f_{i}-\rho u_{i}\right) * \tilde{u}_{i}\right](\mathbf{x}, t) d V=0 \quad(0 \leqq t<\infty),
$$

and (5.7) must hold for every $\tilde{\mathfrak{u}} \in C^{1,2}$ with the foregoing properties. But this fact, together with Lemma 2.1 implies the validity of (4.4). Next let $\tilde{R}=[\tilde{\mathbf{u}}, 0,0,0,0,0,0]$, but this time require only that $\tilde{u}$ vanish on $S_{1} \times[0, \infty)$. Then (5.5), (5.6), (4.4), and Lemma 2.2 yield

$$
g *\left(T_{i}-\hat{T}_{i}\right)=0 \quad \text { on } \quad S_{2} \times[0, \infty),
$$

and this result, because of (2.5), (5.1a), implies (3.8b). Now let $R=[0,0,0, \tilde{\theta}, 0,0,0]$, and let $\tilde{\theta}$, together with all of its space derivatives, vanish on $S \times[0, \infty)$. Then, from (5.1), (5.5), (5.6) follows

$$
\int_{V} \frac{1}{T_{0}}\left[g *\left(g^{\prime} * q_{i, i}-h+\rho T_{0} \eta\right) * \tilde{\theta}\right](\mathbf{x}, t) d V=0 \quad(0 \leqq t<\infty),
$$

and (5.9) must hold for every $\tilde{\theta} \in C^{1,0}$ with the aforementioned properties. This, together with Lemma 2.1 , implies (4.5). Then let $\tilde{R}=[0,0,0, \tilde{\theta}, 0,0,0]$, but require only that $\tilde{\theta}$ vanish on $S_{1} \times[0, \infty)$. Then (5.5), (5.6), (4.5), and Lemma 2.2 imply

$$
\frac{1}{T_{0}} g * g^{\prime} *(Q-\hat{Q})=0 \quad \text { on } \quad S_{2} \times[0, \infty),
$$

and this result, with (2.5), (5.1a), yields (3.8d). Now let $\tilde{R}=[0,0,0,0, \tilde{\eta}, 0,0]$, and suppose $\bar{\eta}$ and all its space derivatives vanish on $S \times[0, \infty)$. By (5.5), (5.6), and Lemma 2.1

$$
g *\left(T_{0} \eta / C_{0}-\beta_{i i}^{\prime} e_{i i}-\theta\right)=0 \text { on } V \times[0, \infty) .
$$

This, together with (2.5), (5.1a), (5.3a), implies (3.5c). Now let $\tilde{R}=[0, \tilde{\mathrm{e}}, \mathbf{0}, 0,0,0,0]$, and suppose $\tilde{\mathrm{e}}$ and all of its space derivatives vanish on $S \times[0, \infty)$. Since $R$ and $\tilde{R}$ are admissible, $\tau$ and ẽ are symmetric. Thus (3.4a), (3.4f), (5.5), (5.6), and Lemma 2.1 yield

$$
g *\left(c_{i j k l}^{\prime} e_{k l}-\rho \beta_{i j}^{\prime} \eta-\tau_{i j}\right)=0 \quad \text { on } \quad V \times[0, \infty),
$$

and this fact, in view of (2.5), (5.1a), (3.5c), (5.3), implies (3.5a). Then let $\tilde{R}=[0,0,0$, $0,0,0, \tilde{\boldsymbol{\vartheta}}]$, and suppose $\tilde{\boldsymbol{\vartheta}}$ and all of its space derivatives vanish on $S \times[0, \infty)$. Using (5.5), (5.6), and Lemma 2.1 yields

$$
\frac{1}{T_{0}} g * g^{\prime} *\left(k_{i} \vartheta_{i}+q_{i}\right)=0 \quad \text { on } \quad V \times[0, \infty),
$$

and this result, with (2.5), (5.1a), implies (3.5b). Next select $\tilde{R}=[0,0, \tilde{\tau}, 0,0,0,0]$, 
where $\tilde{\tau}$ and all of its space derivatives vanish on $S \times[0, \infty)$, and using (5.5), (5.6), the symmetry of $\tilde{\tau}$ and e, Lemma $2.1,(2.5)$, and (5.1a), verify that (3.1a) holds. Then let $\widetilde{R}=[\mathbf{0}, \mathbf{0}, \tilde{\tau}, 0,0, \mathbf{0}, \mathbf{0}]$, but now assume only that $\tilde{\tau}$ and its space derivatives vanish on $S_{2} \times[0, \infty)$. Then (5.5), (5.6), (5.1d), (3.1a), Lemma 2.3, (5.1a), and (2.5) imply (3.8a). Then let $\tilde{R}=[0,0,0,0,0, \tilde{\mathrm{q}}, 0]$, where $\tilde{\mathrm{q}}$ and all its space derivatives are assumed to vanish on $S \times[0, \infty)$. Use (5.5), (5.6), Lemma 2.1, (2.5), and (5.1a) to show that (3.1b) holds. Finally, let $\tilde{R}=[\mathbf{0}, \mathbf{0}, \mathbf{0}, \mathbf{0}, \mathbf{0}, \tilde{\mathbf{q}}, \mathbf{0}]$, but insist only that $\tilde{\mathbf{q}}$ and its space derivatives vanish on $\delta_{2} \times[0, \infty)$. Then (5.5), (5.6), (5.1e), (3.1b), Corollary 2.4, (5.1a), and (2.5) imply (3.8c). Therefore $R$ satisfies (3.1), (3.5), (4.4), (4.5), (3.8), and hence from Theorem $4.2 R$ is seen to be a solution of the mixed problem, which completes the proof.

5.2. Theorem. Let $K$ be the set of all admissible states that meet the strain-displacement and thermal gradient-temperature relations (3.1). Let $R=[\mathbf{u}, \mathbf{e}, \tau, \theta, \eta, \mathbf{q}, \boldsymbol{\vartheta}] \in K$ and for each $t \in[0, \infty)$ define the functional $\Theta_{\imath}\{\cdot\}$ on $K$ by

$$
\begin{aligned}
\Theta_{i}\{R\}= & \int_{V}\left[g * \tau_{i i} * e_{i i}\right](\mathbf{x}, t) d V-\int_{V} \rho(\mathbf{x})[g * \eta * \theta](\mathbf{x}, t) d V \\
& -\frac{1}{2} \int_{V} \chi_{i j k l}^{\prime}(\mathbf{x})\left[g * \tau_{i j} * \tau_{k l}\right](\mathbf{x}, t) d V-\int_{V} \rho(\mathbf{x}) \alpha_{i j}^{\prime}(\mathbf{x})\left[g * \eta * \tau_{i j}\right](\mathbf{x}, t) d V \\
& +\frac{1}{2} \int_{V} \frac{T_{0} \rho(\mathbf{x})}{C_{\tau}(\mathbf{x})}[g * \eta * \eta](\mathbf{x}, t) d V+\frac{1}{2} \int_{V} \rho(\mathbf{x})\left[u_{i} * u_{i}\right](\mathbf{x}, t) d V \\
& +\frac{1}{2} \int_{V} \frac{1}{T_{0}}\left[g * g^{\prime} * q_{i} * \vartheta_{i}\right](\mathbf{x}, t) d V+\frac{1}{2} \int_{V} \frac{\lambda_{i j}(\mathbf{x})}{T_{0}}\left[g * g^{\prime} * q_{i} * q_{i}\right](\mathbf{x}, t) d V \\
& -\int_{V}\left[f_{i} * u_{i}\right](\mathbf{x}, t) d V+\int_{V} \frac{1}{T_{0}}[g * h * \theta](\mathbf{x}, t) d V \\
& +\int_{S_{1}}\left[g *\left(\hat{u}_{i}-u_{i}\right) * T_{i}\right](\mathbf{x}, t) d S-\int_{S_{\mathbf{s}}}\left[g * \hat{T}_{i} * u_{i}\right](\mathbf{x}, t) d S \\
& +\int_{s_{1}} \frac{1}{T_{0}}\left[g * g^{\prime} *(\hat{\theta}-\theta) * Q\right](\mathbf{x}, t) d S-\int_{s_{\mathbf{s}}} \frac{1}{T_{0}}\left[g * g^{\prime} * \hat{Q} * \theta\right](\mathbf{x}, t) d S,(5.14)
\end{aligned}
$$

where $\alpha_{i j}^{\prime}(\mathbf{x})$ and $\chi_{i j k l}^{\prime}(\mathbf{x})$ are given by (4.9). Then

$$
\delta \Theta_{t}\{R\}=0 \quad \text { over } \quad K \quad(0 \leqq t<\infty)
$$

if and only if $R$ is a solution of the mixed problem.

Proof. Let $\tilde{R}=[\tilde{\mathbf{u}}, \tilde{\mathbf{e}}, \tilde{\boldsymbol{\tau}}, \tilde{\theta}, \tilde{\eta}, \tilde{\mathrm{q}}, \tilde{\boldsymbol{\vartheta}}]$ be an admissible state, and suppose in addition that $R+\lambda \tilde{R} \in K$ for every scalar $\lambda$. This latter condition is equivalent to the requirement that $\tilde{R} \in K$. Then, from (5.14), (2.7), (3.1), (3.4), the properties of the convolution, and the divergence theorem, follows

$$
\begin{aligned}
\delta_{\tilde{L}} \Theta_{\iota}\{R\}= & \int_{V}\left[g *\left(e_{i j}-\chi_{i j k l}^{\prime} \tau_{k l}-\rho \alpha_{i j}^{\prime} \eta\right) * \tilde{\tau}_{i j}\right](\mathbf{x}, t) d V \\
& +\int_{V} \rho(\mathrm{x})\left[g *\left(\frac{T_{0} \eta}{C_{\tau}}-\alpha_{i j}^{\prime} \tau_{i i}-\theta\right) * \tilde{\eta}\right](\mathbf{x}, t) d V \\
& +\int_{V} \frac{1}{T_{0}}\left[g * g^{\prime} *\left(\vartheta_{i}+\lambda_{i i} q_{j}\right) * \tilde{q}_{i}\right](\mathbf{x}, t) d V
\end{aligned}
$$




$$
\begin{aligned}
& -\int_{V}\left[\left(g * \tau_{i, i, j}+f_{i}-\rho u_{i}\right) * \tilde{u}_{i}\right](\mathbf{x}, t) d V \\
& -\int_{V} \frac{1}{T_{0}}\left[g *\left(g^{\prime} * q_{i, i}-h+\rho T_{0} \eta\right) * \tilde{\theta}\right](\mathbf{x}, t) d V \\
& +\int_{S_{2}}\left[g *\left(\hat{u}_{i}-u_{i}\right) * \tilde{T}_{i}\right](\mathbf{x}, t) d S+\int_{S_{2}}\left[g *\left(T_{i}-\hat{T}_{i}\right) * \tilde{u}_{i}\right](\mathbf{x}, t) d S \\
& +\int_{S_{1}} \frac{1}{T_{0}}\left[g * g^{\prime} *(\hat{\theta}-\theta) * \tilde{Q}\right](\mathbf{x}, t) d S+\int_{s_{2}} \frac{1}{T_{0}}\left[g * g^{\prime} *(Q-\hat{Q}) * \tilde{\theta}\right](\mathbf{x}, t) d S
\end{aligned}
$$

If $R$ is a solution of the mixed problem, then (5.16), because of Theorem 4.3, yields

$$
\delta_{\tilde{R}} \Theta_{t}\{R\}=0, \quad 0 \leqq t<\infty, \quad \text { for every } \tilde{R} \in K,
$$

which implies (5.15). On the other hand (5.16), (5.17), Lemma 2.1, Lemma 2.2, Lemma 2.3, Corollary 2.4, (2.5), (3.1), and Theorem 4.3 imply that $R$ is a solution to the mixed problem, and the theorem is proved.

Another form of variational principle can be developed by requiring more stringent conditions on the admissible states. Analogous to the notion of a kinematically admissible state as used by Gurtin [5], a kinematically and thermally admissible state is introduced as a state that meets the strain-displacement and thermal gradient-temperature relations (3.1), the constitutive relations and equation of state (3.5), and the displacement and temperature boundary conditions (3.8a), (3.8c). The variational principle based upon such states represents an extension of the theorem of minimum potential energy.

5.3. Theorem. Let $K$ be the set of all kinematically and thermally admissible states. Let $R=[\mathbf{u}, \mathbf{e}, \tau, \theta, \eta, \mathbf{q}, \boldsymbol{\vartheta}] \in K$, and for each $t \in[0, \infty)$ define the functional $\Phi_{t}\{\cdot\}$ on $K$ by

$$
\begin{aligned}
\Phi_{t}\{R\}= & \frac{1}{2} \int_{V}\left[g * \tau_{i j} * e_{i j}\right](\mathbf{x}, t) d V-\frac{1}{2} \int_{V} \rho(\mathbf{x})[g * \eta * \theta](\mathbf{x}, t) d V \\
& +\frac{1}{2} \int_{V} \rho(\mathbf{x})\left[u_{i} * u_{i}\right](\mathbf{x}, t) d V+\frac{1}{2} \int_{V} \frac{1}{T_{0}}\left[g * g^{\prime} * q_{i} * \vartheta_{i}\right](\mathbf{x}, t) d V \\
& -\int_{V}\left[f_{i} * u_{i}\right](\mathbf{x}, t) d V+\int_{V} \frac{1}{T_{0}}[g * h * \theta](\mathbf{x}, t) d V \\
& -\int_{S_{3}}\left[g * \hat{T}_{i} * u_{i}\right](\mathbf{x}, t) d S-\int_{\delta_{2}} \frac{1}{T_{0}}\left[g * g^{\prime} * \hat{Q} * \theta\right](\mathbf{x}, t) d S
\end{aligned}
$$

Then

$$
\delta \Phi_{t}\{R\}=0 \quad \text { over } K \quad(0 \leqq t<\infty)
$$

if and only if $R$ is a solution to the mixed problem.

Proof. Let $\tilde{R}=[\tilde{\mathbf{u}}, \tilde{\mathbf{e}}, \tilde{\boldsymbol{\tau}}, \tilde{\theta}, \tilde{\eta}, \tilde{\mathbf{q}}, \tilde{\boldsymbol{\vartheta}}]$ be an admissible state and suppose that

$$
R+\lambda \tilde{R} \in K \text { for every scalar } \lambda \text {. }
$$

This latter condition is equivalent to the requirement that $\tilde{R}$ meets (3.1), (3.5), with 


$$
\tilde{u}_{i}=0 \quad \text { on } \quad S_{1} \times[0, \infty)
$$

and

$$
\tilde{\theta}=0 \quad \text { on } \quad S_{1} \times[0, \infty) .
$$

Next use (5.18), (2.7), (3.1), (3.4), (3.5), (5.21), the properties of the convolution, and the divergence theorem to verify that

$$
\begin{aligned}
\delta_{\tilde{R}} \Phi_{t}\{R\}= & -\int_{V}\left[\left(g * \tau_{i j, i}+f_{i}-\rho u_{i}\right) * \tilde{u}_{i}\right](\mathbf{x}, t) d V \\
& -\int_{V} \frac{1}{T_{0}}\left[g *\left(g^{\prime} * q_{i, i}-h+\rho T_{0} \eta\right) * \tilde{\theta}\right](\mathbf{x}, t) d V \\
& +\int_{S_{2}}\left[g *\left(T_{i}-\hat{T}_{i}\right) * \tilde{u}_{i}\right](\mathbf{x}, t) d S \\
& +\int_{s_{2}} \frac{1}{T_{0}}\left[g * g^{\prime} *(Q-\hat{Q}) * \tilde{\theta}\right](\mathbf{x}, t) d S \quad(0 \leqq t<\infty),
\end{aligned}
$$

for every $\tilde{u}_{i} \in C^{1,2}$ and $\tilde{\theta} \in C^{1,0}$ which meet (5.21). First suppose $R$ is a solution to the mixed problem. Then (5.22), by virtue of Theorem 4.3, implies (5.19). On the other hand (5.19), (5.22), Lemma 2.1, Lemma 2.2, (2.5), (3.1), (3.5), and Theorem 4.3 imply that $R$ is a solution of the mixed problem. This completes the proof.

6. Variational characterization of displacements and temperature. By an admissible displacement and temperature field is meant an ordered pair $[\mathbf{u}, \theta]$ such that the vectorvalued function $\mathfrak{u} \in C^{1,2}$ and the scalar-valued function $\theta \in C^{1,0}$. The linear space of interest here will be the set of all admissible displacement and temperature fields By a kinematically and thermally admissible displacement and temperature field is meant an admissible displacement and temperature field satisfying the boundary conditions (3.8a) and (3.8c) on the displacement and temperature. It follows that, if $[\mathbf{u}, \mathbf{e}, \boldsymbol{\tau}, \theta, \eta, \mathbf{q}, \boldsymbol{\vartheta}]$ is a kinematically and thermally admissible state, then $[\mathbf{u}, \theta]$ is a kinematically and thermally admissible displacement and temperature field. Conversely, the latter assertion implies the former when $\mathbf{e}, \boldsymbol{\vartheta}, \tau, \eta$, and $\mathbf{q}$ are defined through (3.1) and (3.5). Thus the following corollary to Theorem 5.3 may be stated.

6.1. Corollary. Let $K$ be the set of all kinematically and thermally admissible displacement and temperature fields. Let $[\mathfrak{u}, \theta] \in K$ and for each $t \in[0, \infty)$ define the functional $\varphi_{t}\{\cdot\}$ on $K$ through

$$
\begin{aligned}
\varphi_{t}\{\mathbf{u}, \theta\}= & \frac{1}{2} \int_{V} c_{i j k l}(\mathbf{x})\left[g * u_{i, i} * u_{k, l}\right](\mathbf{x}, t) d V \\
& +\frac{1}{2} \int_{V} \rho(\mathbf{x})\left[u_{i} * u_{i}\right](\mathbf{x}, t) d V-\int_{V} \beta_{i j}(\mathbf{x})\left[g * u_{i, i} * \theta\right](\mathbf{x}, t) d V \\
& -\frac{1}{2} \int_{V} \frac{\rho(\mathbf{x}) C_{e}(\mathbf{x})}{T_{0}}[g * \theta * \theta](\mathbf{x}, t) d V-\frac{1}{2} \int_{V} \frac{k_{i j}(\mathbf{x})}{T_{0}}\left[g * g^{\prime} * \theta_{, i} * \theta_{, i}\right](\mathbf{x}, t) d V \\
& -\int_{V}\left[f_{i} * u_{i}\right](\mathbf{x}, t) d V+\int_{V} \frac{1}{T_{0}}[g * h * \theta](\mathbf{x}, t) d V \\
& -\int_{S_{\mathbf{s}}}\left[g * \hat{T}_{i} * u_{i}\right](\mathbf{x}, t) d S-\int_{s_{2}} \frac{1}{T_{0}}\left[g * g^{\prime} * \hat{Q} * \theta\right](\mathbf{x}, t) d S
\end{aligned}
$$


Then

$$
\delta \varphi_{t}\{\mathbf{u}, \theta\}=0 \quad \text { over } K \quad(0 \leqq t<\infty)
$$

if and only if $\mathbf{u}$ and $\theta$ are displacement and temperature fields corresponding to a solution of the mixed problem of coupled thermoelasticity.

7. Variational characterization of stress and heat flux. By an admissible stress and heat flux field is meant an ordered pair [ $\tau, \mathrm{q}]$ such that the symmetric second-order tensorvalued function $\tau \in C^{2,0}$ and the vector-valued function $q \in C^{2,0}$. The linear space basic to the development here is the set of all admissible stress and heat flux fields. By a dynamically and energetically admissible stress and heat flux field is meant an admissible stress and heat flux field satisfying the boundary conditions (3.8b) and (3.8d) on the traction vector and normal heat flux.

7.1. Theorem. Let $K$ be the set of all dynamically and energetically admissible stress and heat flux fields. Let $R=[\tau, \mathrm{q}] \in K$ and for each $t \in[0, \infty)$ define the functional $\Lambda_{t}\{\cdot\}$ on $K$ through

$$
\begin{aligned}
\Lambda_{\imath}\{R\}= & \frac{1}{2} \int_{V} \chi_{i j k l}^{\prime}(\mathbf{x})\left[\tau_{i j} * \tau_{k l}\right](\mathbf{x}, t) d V+\int_{V} \frac{\alpha_{i j}^{\prime}(\mathbf{x})}{T_{0}}\left[\tau_{i j} *\left(h-g^{\prime} * q_{m, m}\right)\right](\mathbf{x}, t) d V \\
& -\frac{1}{2} \int_{V} \frac{\lambda_{i j}(\mathbf{x})}{T_{0}}\left[g^{\prime} * q_{i} * q_{i}\right](\mathbf{x}, t) d V+\frac{1}{2} \int_{V} \frac{1}{\rho(\mathbf{x})}\left[g * \tau_{i j, i} * \tau_{i m, m}\right](\mathbf{x}, t) d V \\
& -\frac{1}{2} \int_{V} \frac{1}{\rho(\mathbf{x}) C_{\tau}(\mathbf{x}) T_{0}}\left[g^{\prime} * g^{\prime} * q_{i, i} * q_{m, m}\right](\mathbf{x}, t) d V \\
& +\int_{V} \frac{1}{\rho(\mathbf{x})}\left[f_{i} * \tau_{i j, i}\right](\mathbf{x}, t) d V+\int_{V} \frac{1}{\rho(\mathbf{x}) C_{\tau}(\mathbf{x}) T_{0}}\left[g^{\prime} * h * q_{i, i}\right](\mathbf{x}, t) d V \\
& -\int_{S_{1}}\left[\hat{u}_{i} * T_{i}\right](\mathbf{x}, t) d S-\int_{S_{1}} \frac{1}{T_{0}}\left[g^{\prime} * \hat{\theta} * Q\right](\mathbf{x}, t) d S
\end{aligned}
$$

where $\alpha_{i j}^{\prime}(\mathbf{x})$ and $\chi_{i j k l}^{\prime}(\mathbf{x})$ are given by (4.9). Then

$$
\delta \Lambda_{t}\{R\}=0 \quad \text { over } K \quad(0 \leqq t<\infty)
$$

if and only if $\tau$ and $\mathbf{q}$ are stress and heat flux fields corresponding to a solution of the mixed problem of coupled thermoelasticity.

Proof. Let $\tilde{R} \in K$ so that

$$
\tilde{T}_{i}=\tilde{\tau}_{i j} n_{i}=0 \quad \text { on } \quad S_{2} \times[0, \infty)
$$

and

$$
\tilde{Q}=\tilde{q}_{i} n_{i}=0 \quad \text { on } \quad S_{2} \times[0, \infty) .
$$

It follows that $R+\lambda \tilde{R} \in K$ for every scalar $\lambda$. Then, by (7.1), (3.4b), (3.4d), (3.4e), (2.7), (7.3), the properties of the convolution, the symmetry of $\tau$, and the divergence theorem, 


$$
\begin{aligned}
& \delta_{\tilde{R}} \Lambda_{t}\{R\}=\int_{V}\left\{\left[\chi_{i j k l}^{\prime} \tau_{k l}+\frac{\alpha_{i j}^{\prime}}{T_{0}^{\prime}}\left(h-g^{\prime} * q_{m, m}\right)-\left(\frac{1}{\rho}\left[g * \tau_{(i m, m}+f_{(i}\right]\right)_{, i)}\right] * \tilde{\tau}_{i j}\right\}(\mathbf{x}, t) d V \\
& +\int_{V} \frac{1}{T_{0}} g^{\prime} *\left\{\left[\left(\alpha_{k l}^{\prime} \tau_{k l}-\frac{1}{\rho C_{\tau}}\left[h-g^{\prime} * q_{m, m}\right]\right)_{, i}-\lambda_{i j} q_{i}\right] * \tilde{q}_{i}\right\}(\mathbf{x}, t) d V \\
& +\int_{S_{i}}\left\{\left[\frac{1}{\rho}\left(g * \tau_{i j, i}+f_{i}\right)-\hat{u}_{i}\right] * \tilde{T}_{i}\right\}(\mathbf{x}, t) d S \\
& +\int_{S_{i}} \frac{1}{T_{0}} g^{\prime} *\left\{\left[\frac{1}{\rho C_{\tau}}\left(h-g^{\prime} * q_{i, i}\right)-\alpha_{i j}^{\prime} \tau_{i j}-\hat{\theta}\right] * \tilde{Q}\right\}(\mathbf{x}, t) d S, \quad 0 \leqq t<\infty,(7.4)
\end{aligned}
$$

for every $\tilde{R} \in K$. If $R$ corresponds to a solution of the mixed problem then (7.4) and Theorem 4.5 yield (7.2). On the other hand (7.2), (7.3), (7.4), (3.4), the symmetry of $\tau$, Lemma 2.1, Lemma 2.3, Corollary 2.4, (2.5), (5.1), and Theorem 4.5 imply that $R$ is a solution to the mixed problem. This completes the proof.

In closing we note that extensions of these theorems to a theory of time-invariant thermoviscoelasticity, in which the mechanical properties are independent of temperature, are easily obtained. Because of the strong thermal dependence of the relaxation moduli of a real viscoelastic material, such a theory is felt to be of little significance.

\section{REFERENCES}

1. M. A. Biot, Thermoelasticity and irreversibie thermodynamics, J. Appl. Phys. 27, 3, 240 (1956)

2. G. Herrmann, On variational principles in thermoelasticity and heat conduction, Q. Appl. Math. 21, 2, $151(1963)$

3. Bao-Lian', Fu, The generalized varialional principles of thermoelasticity, Scientia Sinica 13, 9, 1507 (1964) (in Russian)

4. M. Ben-Amoz, On a variational theorem in coupled thermoelasticity, Trans. ASME, J. Appl. Mech. 32, 4, 943 (1965).

5. M. E. Gurtin, Variational principles for linear elastodynamics, Arch Rat. Mech. Anal. 16, 1, 34 (1964)

6. M. E. Gurtin, Variational principles for linear initial-value problems, Q. Appl. Math. 22, 3, 252 (1964)

7. J. Mikusinski, Operational calculus, Pergamon Press, New York, 1959

8. O. D. Kellogg, Foundations of potential theory, F. Ungar Publishing Co., New York, 1929

9. E. C. Titchmarsh, The zeros of certain integral functions, Proc. London Math. Soc. 25, 4, 283 (1926)

10. J. Ignaczak, A completeness problem for stress equations of motion in the linear elasticity theory, Arch. Mech. Stos. 15, 2, 225 (1963)

11. Hai-Chang $\mathrm{Hu}, \mathrm{On}$ some variational principles in the theory of elasticity and the theory of plasticity, Scientia Sinica 4, 1, 33 (1955)

12. K. Washizu, On the variational principles of elasticity and plasticity, ASRL TR 25-18, Massachusetts Institute of Technology

13. E. Hellinger, Die allgemeinen ansätze der Mechanik der Kontinua, Encyclopädie der Mathematischen Wissenschaften, 4, parts 4 and 5, 654 (1914)

14. E. Reissner, On a variational theorem in elasticity, J. Math. Phys. 29, 2, 90 (1950)

15. A. E. H. Love, A treatise on the mathematical theory of elasticity, 4th Ed., Cambridge University Press, 1927 